\title{
Primary malignant melanoma involving the anorectum
}

\author{
ERIC A. GOLDSMITH, Do \\ EDWARD A. GOTFRIED, DO, FACOS \\ J. HARRIS JOSEPH, DO
}

Primary malignancy of the anorectum is an uncommon neoplasm with a poor prognosis. The typical patient presents in the second decade of life, is white, with a chief complaint of rectal bleeding. At the time of diagnosis, most patients will already have metastatic disease. The appropriate surgical treatment remains controversial.

Primary malignant melanoma of the anorectum was first reported by Moore ${ }^{1}$ in 1857 . A comparatively rare neoplasm, this lesion comprises about $1 \%$ of all malignancies of the anorectal region. ${ }^{2,3}$ However, after the skin and retina, the anorectum is the third most common site of melanoma, ${ }^{2,4}$ which occurs with a frequency of approximately one for every eight epidermoid tumors of the anus and every 250 adenocarcinomas of the rectum. ${ }^{5,6}$ Malignant melanoma of the anorectum is a latediagnosed, usually already metastasized disease that carries a dispiriting prognosis, with an overall five-year survival rate of only $10 \%$. $^{7,8}$

Several authors $\mathrm{s}^{3,7,9}$ alternately have reported a male or female predominance in anorectal melanoma. A review of 178 cases reported in the past two decades revealed a $1.5: 1$ female-to-male ratio. ${ }^{2-13,17-19}$ Therefore, no significant sex predilection has been demonstrated. The average age for this disease is 60 years, with a range from 22 to 84 years. Although the statistics reveal malignant melanoma of the anorectum to be by far a neoplasm of whites, there are several reported cases involving blacks, ${ }^{10,11}$ Orientals, ${ }^{12}$ and others. ${ }^{4,13}$ Tumor sizes ranged from $<1 \mathrm{~cm}$ to $>7 \mathrm{~cm}$, with most lesions reported to be between $2 \mathrm{~cm}$ and $6 \mathrm{~cm}$.

Rectal bleeding is the most common presenting complaint; anal mass and pain are the next most frequent symptoms. Unfortunately, the character of the bleeding is easily mistaken for hemorrhoids, which is partly to blame for delays in diagnosis. Some authors ${ }^{7,12}$ have reported one to five years of symptoms before correct diagnosis was made. On the whole, however, three to eight months is the average duration of symptoms before malignant melanoma is diagnosed. . $^{-10,17-19}$

The diagnosis of melanoma depends upon the presence of certain histiologic features. The large epithelioid cells may be spindle shaped or sphenoidal and contain a varied amount of melanin pigment. Pleomorphism usually is marked (Fig 1). Junctional activity (Fig 2) makes the diagnosis easy, but ulceration may destroy this feature. Amelanotic tumors in particular may show clustering of cells. It is interesting to note that $16 \%$ to $41 \%$ of anorectal melanomas are amelanotic. ${ }^{10,11,17}$ Of those authors with an opinion, all agree that the histologic type contributed nothing as a prognostic indicator for survival.

We have recently treated two patients with primary malignant melanoma of the anorectum. Two different treatment modalities were employed.

\section{Report of cases}

Case 1

A 62-year-old white man presented with a chief complaint of bright red rectal bleeding. The patient also complained of constipation, which had worsened recently. No other constitutional complaints were offered. Physical examination was positive for a protruding, palpable mass from the anus; this was bluish-black in color and tender. No active bleeding was noted. A small, firm lesion also was palpated in the anterior abdominal wall, just to the left of the umbilicus. There was no lymphadenopathy.

Laboratory studies included complete blood count and serum electrolyte and liver enzyme determinations, all 


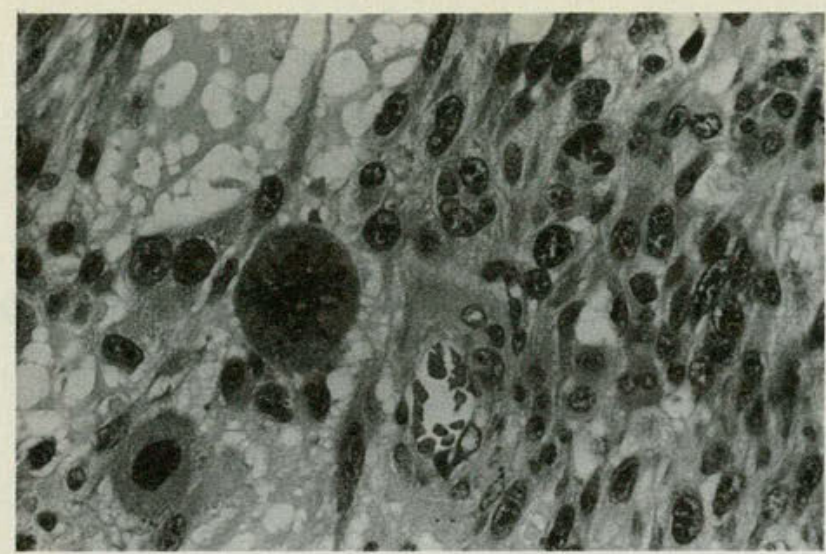

Fig 1. Monstrous, abnormal mitosis is demonstrated. Other melanoma cells have a spindle shape, irregular nuclei, and prominent, multiple nucleoli (hematoxylin and eosin stain, original magnification $\times 40$ ).

of which had normal results. Liver and spleen scanning and barium enema failed to reveal any pathologic condition.

Wide excision of the anal mass and removal of the lesion of the anterior abdominal wall were performed. The polypoid mass removed from the anus measured approximately $5 \mathrm{~cm} \times 1.5 \mathrm{~cm}$. Microscopic sections demonstrated large cells, with large, prominent nuclei and abundant dark, coarse, pigmented granules contained in their cytoplasm (Fig 3). Identified zones of junctional activity and ulceration were found in the squamous epithelium. Vascular and perineural space invasion was noted, and special staining for melanin was positive. The abdominal wall lesion was grossly melanotic and represented a metastatic deposit.

Computed tomographic (CT) scanning of the brain, abdomen, and chest revealed bilateral pulmonary parenchymal metastatic disease as well as metastasis to the brain. The patient was discharged on a course of chemotherapy with dacarbazine (DTIC) and hydrocortisone. He had several hospital readmissions for neurologic and bleeding sequelae of his disease. Serial serum lactic dehydrogenase values were documented to a high of 2,998 units at $30^{\circ} \mathrm{C}$. Five months after diagnosis, the patient died.

\section{Case 2}

A 65-year-old black man was seen as an outpatient for complaint of a protruding rectal mass, bloody discharge, and occasional rectal pain. Physical examination was significant for a tender, protruding mass from the anal verge, with proximal extensions for $6 \mathrm{~cm}$. There were no palpable inguinal nodes. On admission, laboratory studies, including complete blood count and liver enzyme and serum electrolyte determinations, were unremarkable. Abdominal and pelvic CT scanning disclosed a softtissue density within the anorectum and minimal hepatomegaly.

Biopsy revealed a polypoid-shaped mass, which meas-

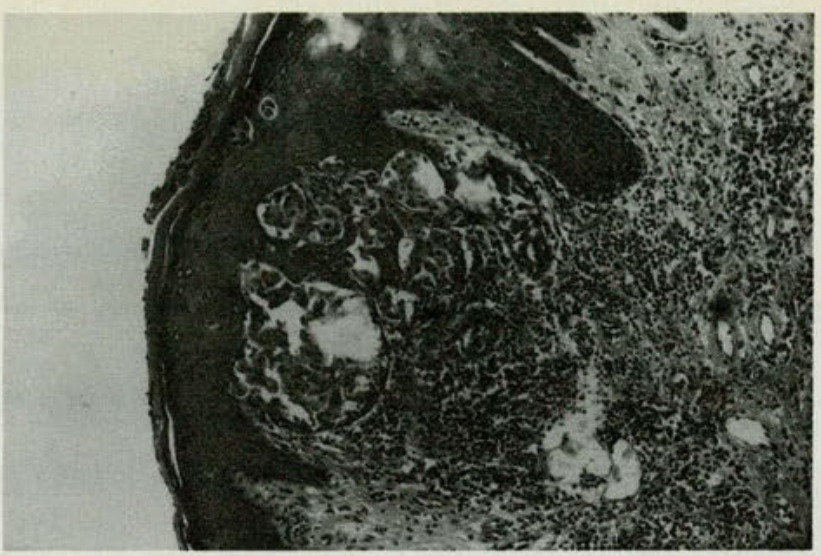

Fig 2. Squamous epithelium from the anal region is infiltrated by nests of rounded, epithelioid melanoma cells in a pagetoid pattern. The base of the lesion shows a prominent lymphocytic reaction (hematoxylin and eosin stain, original magnification $\times 40)$.

ured $4.8 \mathrm{~cm} \times 3.5 \mathrm{~cm}$. The tissue was described as graytan in color. Microscopic sections showed ulcerated mucosa replaced by infiltrating neoplasm, which was composed of elongated, spindle-type cells with enlarged nuclei (Fig 4). Prominent melanocytic junctional activity was seen in the intact portions of squamous epithelium. Immunoperoxidase staining was positive for uptake that represented malignant melanoma.

Miles' abdominal-perineal resection was performed. Two mesenteric nodes were submitted with the specimen; both were negative for malignancy. The resected anorectum showed no evidence of residual tumor. The patient was discharged in good condition, and a followup schedule was arranged. $\mathrm{He}$ is alive and well eight months after initial diagnosis.

\section{Discussion}

It has generally been accepted that melanoma of the anorectum arises from differentiated pigment cells called melanocytes, which are closely related to nerve tissue as part of a placoid neuroectoderm or neural crest. ${ }^{14,15} \mathrm{~A}$ detailed study ${ }^{16}$ of this region could not document melanocytes located above the anal pecten. Melanoma of the anal region will spread in the submucosa, without causing mucosal disruption, and then will reappear more proximally in the rectum. These rectal lesions, then, actually represent spread of a primary anal lesion. ${ }^{8,10,17}$

Malignant melanoma also disseminates via the blood and lymph channels. Lymphatic spread can be described as superficial (to the inguinal lymph nodes), lateral (to the iliac and obturator nodes), or deep (to the inferior mesenteric and aortic groups). It has been theorized that pelvic nodal groups are the first to become involved..$^{10,13} \mathrm{As}$ these become replaced by tumor, lymph flow be- 


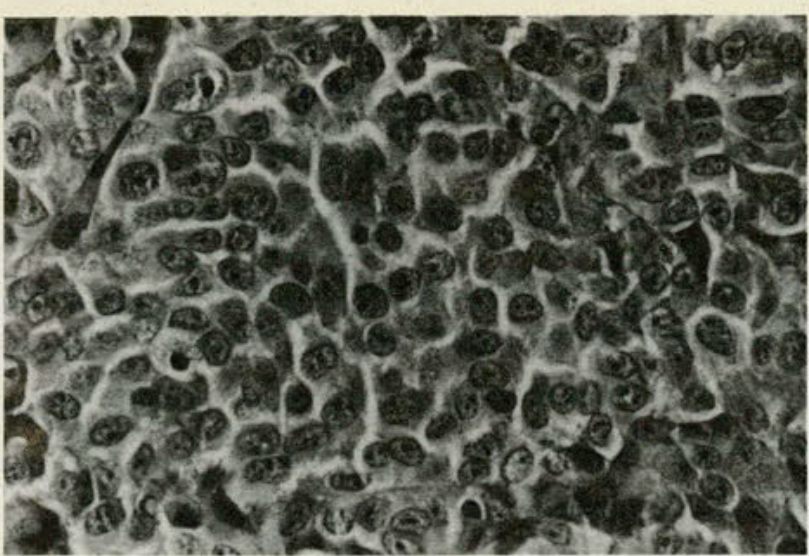

Fig 3. Microscopic section of anal mass demonstrates rounded-topolygonal, epithelioid-appearing cells with large, irregular nuclei and prominent eosinophilic nucleoli (case 1). Darkly staining melanin pigment is seen in some of the cells (hematoxylin and eosin stain, original magnification $\times 400$ ).

comes altered, so that the inguinal nodes become involved later. Mesenteric nodes become involved before the inguinal nodes because melanoma tends to invade the rectal mucosa early. ${ }^{4,8,13}$ Hematogenous spread occurs via the inferior mesenteric vein to the portal drainage and by the internal pudendal vein to the systemic circulation. The resultant metastatic disease involves the brain, liver, bone, lung, and skin.

The search for the best surgical treatment of malignant melanoma of the anorectum is far from finished. Some authors ${ }^{2,4,8,10}$ have shown that patients treated with abdominoperineal resection of local excision produced no statistically significant difference in survival rates. Others ${ }^{3,7,13,18}$ suggested that a more radical approach of Miles' resection (in some cases including bilateral groin dissection or en bloc pelvic node dissection) was responsible for five-year survivors in their series. A review ${ }^{19}$ of 165 cases disclosed 85 abdominoperineal resections, with an average survival time of 32.4 months; there were 11 five-year survivors. Eighty patients were treated by local excision; the average survival time was 23.7 months, and there were six five-year survivors.

Close inspection of the data shows a very definite correlation between presence or absence of metastatic disease and long-term survival. Fiveyear survivors had shown no evidence of metastatic spread at the time of surgery and were classified as having stage I disease.

Because the anorectum does not contain the classic dermatologic layers, Clark's classification of tumor invasion cannot be applied. Most authors also have discarded the application of Breslow's ${ }^{20}$ predictive value of tumor thickness. One series ${ }^{8}$ of 36

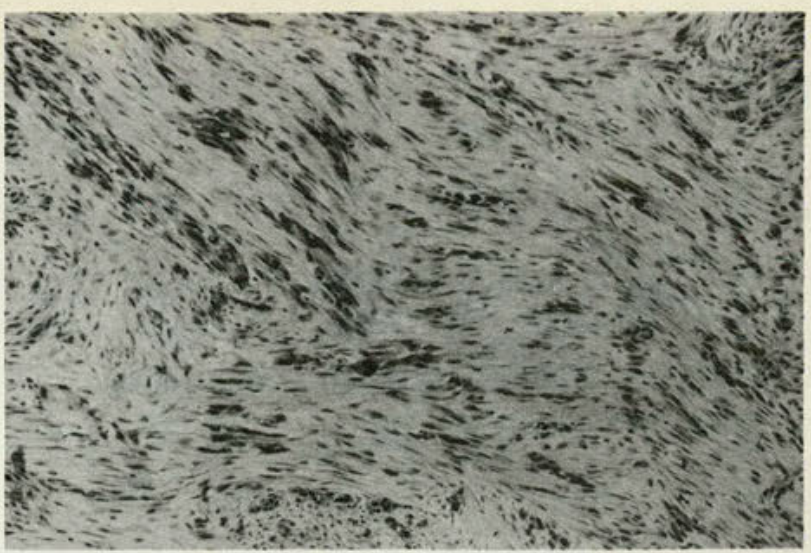

Fig 4. Predominantly spindle cells are seen, which demonstrates the "neurotized" characteristics of this type of melanoma (case 2) (hematoxylin and eosin stain, original magnification $\times 40$ ).

patients compared radical surgery (abdominoperineal resection with or without nodal dissection) to conservative surgery (wide excision with or without nodal dissection). The only five-year survivors were those who underwent the most aggressive surgical therapy. Three of four of these patients were classified as having stage I disease, and all four had lesions that were $<2 \mathrm{~mm}$ in thickness. Thus, this study supports the correlation of clinical stage to survival time and extends the concept of tumor thickness as an indicator for survival of anorectal melanoma.

To date, there has been no documentation of benefit from chemotherapy or radiation therapy in the curative management of anorectal melanoma. ${ }^{21}$ There are scattered reports that radiation may provide some palliation for metastatic disease. ${ }^{6}$ The use of adjuvant chemotherapy is still unclear; DTIC has been used with some limited success in treating disseminated melanoma. ${ }^{12,17}$

In reviewing 230 cases of malignant melanoma of the anorectum, we were able to find only 12 (5.2\%) five-year survivors. ${ }^{2-13,17-19,22-25}$ The experience at Memorial Sloan-Kettering Cancer Center perhaps underscores how little we have progressed in the treatment of this disease: Patients treated after 1950 had the same median survival rate as those treated prior to that date. ${ }^{8}$

\section{Conclusions}

Malignant melanoma of the anorectum is a relatively uncommon disease that has no sex predilection. The most common signs and symptoms are rectal bleeding, pain, and mass. Treatment options remain controversial. Local excision provides a comparable survival rate to a more radical procedure. 
Abdominoperineal resection samples regional lymph nodes for proper staging and shows less tendency for local recurrence. Neither chemotherapy nor radiation therapy has been effective in curative management of melanoma of the anorectum.

Improvement of survival from this disease must depend ultimately on more aggressive evaluations of even trivial symptoms with digital examination and rigid or flexible endoscopic examination, biopsy of any suspect lesions, and complete staging workup. Stage I tumors and those found to be < $2 \mathrm{~mm}$ in thickness tend toward favorable prognosis.

J. Harris Joseph, DO, supplied the data for the two case reports.

1. Moore WD: Recurrent melanoma of the anus after previous removal from the verge of the anus. Lancet 1857;1:290.

2. Husa A, Hockerstedt K: Anorectal malignant melanoma: A report of fourteen cases. Acta Chir Scand 1974;140:68-72.

3. Pack GT, Oropeza R: A comparative study of melanoma and epidermoid carcinoma of the anal canal: A review of 20 melanomas and 29 epidermoid carcinomas (1930-1965). Dis Colon Rectum 1967;10:161176.

4. Mason JK, Helwig EB: Anorectal melanoma. Cancer 1966;19:39-50.

5. Morson BC, Volkstaedt H: Malignant melanoma of the anal canal. $J$ Clin Pathol 1963;16:126-132.

6. Quan SH, Deddish MR: Noncutaneous melanoma: Malignant melanoma of the anorectum. Cancer 1966;16:111-114.

7. Chiu YS, Unni KF, Beart RW Jr: Malignant melanoma of the anorectum. Dis Colon Rectum 1980;23:122-124.

8. Wanebo H, Woodruff JM, Farr GH, et al: Anorectal melanoma. Cancer 1981;47:1891-1900.

9. Braastad FW, Dockerty MB: Melano-epithelioma of the anus and rectum. Surgery 1949;25:82-90.
10. Cooper PH, Mills SE, Allen MS, Jr: Malignant melanoma of the anus: Report of 12 patients and analysis of 255 additional cases. Colon Rectum 1982;25:693-703.

11. Quinn D, Selab C: Malignant melanoma of the anus in a Negro: Report of a case and review of the literature. Dis Colon Rectum 1977;20:627-631.

12. Boey J, Choi TK, Wong J, et al: The surgical management of anorectal malignant melanoma. Aus NZ Surg 1981;51:132-136.

13. Baskies AM, Sugarbaker EJ, Chretien PB, et al: Anorectal melanoma: The role of posterior pelvic exenteration. Dis Colon Rectum 1982;25:772-777.

14. Masson P: Pigment cells in man. New York Acad Sci 1948;4:15-47. 15. Rawles ME: Origin of pigment cells form the neural chest in mouse embryo. Physiol Zool 1947;20:248-266.

16. Walls, EW: Observations on the microscopic anatomy of the human anal canal. Br J Surg 1958; 45:504-512.

17. Alexander RM, Cone LA: Malignant melanoma of the rectal ampulla. Report of 6 cases and review of the literature. Dis Colon Rectum 1977;20:53-55.

18. Hickey M, Magee D: Malignant melanoma of the anal canal. Ir Med $J$ 1982;75:83.

19. Bolivar JC, Harris JW, Branch W, et al: Melanoma of the anorectal region. Surg Gynec Obstet 1982,154:337-341.

20. Breslow A: Thickness, cross-sectional areas and depth of invasion in the prognosis of cutaneous melanoma. Ann Surg 1970;172:902-908.

21. Angeras U, Johnson N, Jonsson PE: Primary anorectal malignant melanoma. J Surg Oncol 1983;22:261-264.

22. Abbas JS, Karakousis CR, Holyoke ED: Anorectal melanoma: Clinical features, recurrence and patient survival. Int Surg 1980;65:423-426. 23. Herrera L, Glassman CT: Maligant melanoma of the anorectum. Del Med J 1979;51:323-324.

24. Mulder SPM, Veldsman JJ: Malignant melanoma of the anal canal. Afr Med $J$ 1985;67:101-102.

25. Quan SH: Anal and para-anal tumors. Surg Clin North Am 1978;58:591-603.

From the Department of Surgery, Metropolitan Hospital, Springfield Division, Springfield, Pa.

Reprints requests to Dr Goldsmith, Metropolitan Hospital, Springfield Division, 190 W Sproul Rd, Springfield, PA 19064. 